\title{
Diagrama Entidade-Relacionamento: uma ferramenta para modelagem de dados conceituais em Engenharia de Software
}

\author{
Ratio-Entity Diagram: a tool for conceptual data modeling in Software Engineering
}

Diagrama entidad-relación: una herramienta para el modelado de datos conceptuales en Ingeniería de Software

\author{
Kewry Mariobo Franck \\ ORCID: https://orcid.org/0000-0002-5972-1924 \\ Centro Universitário São Lucas, Brasil \\ E-mail: kewryfranck@gmail.com \\ Robson Fernandes Pereira \\ ORCID: https://orcid.org/0000-0003-0983-7950 \\ Instituto Brasileiro do Meio Ambiente e dos Recursos Naturais Renováveis, Brasil \\ E-mail: Bed_1@ @otmail.com \\ Jerônimo Vieira Dantas Filho \\ ORCID: http://orcid.org/0000-0002-5965-9438 \\ Universidade Federal de Rondônia, Brasil \\ E-mail: jeronimovdantas@gmail.com
}

\begin{abstract}
Resumo
A modelagem de dados é o principal componente de informações conceituais do banco de dados, dentre as técnicas para essa modelagem, a entidade-relacionamento (ER) é abordada nesse trabalho. O objetivo do estudo é abordar as principais informações sobre o modelo entidade-relacionamento como ferramenta para modelagem de dados conceituais. O Modelo Entidade-Relacionamento, e principalmente o diagrama, é uma importante ferramenta durante o desenvolvimento de sistemas, principalmente aqueles mais complexos e difíceis de visualizar sem uma análise mais aprofundada. Além disso, é um modelo conceitual utilizado na Engenharia de Software para descrever as entidades envolvidas em um domínio de negócios, com seus atributos e como elas se relacionam entre si. De modo geral, representa de forma abstrata a estrutura que possuirá o banco de dados da aplicação. O banco de dados poderá conter várias outras entidades, tais como chaves e tabelas intermediárias, que podem fazer sentido no contexto de bases de dados relacionais.
\end{abstract}

Palavras-chave: Banco de dados; Dados relacionais; Modelagem conceitual.

\begin{abstract}
Data modeling is the main component of conceptual information in the database. Among the techniques for this modeling, Ratio-Entity (RE) is addressed in this work. The aimed is to address the main information about the entity-relationship model as a tool for conceptual data modeling. The Ratio-Entity Model, and especially the diagram, is an important tool during the development of systems, especially those more complex and difficult to visualize without further analysis. In addition, it is a conceptual model used in Software Engineering to describe the entities involved in a business domain, with their attributes and how they relate to each other. In general, it represents in an abstract way the structure that will have the application database. The database may contain several other entities, such as keys and intermediate tables, that may make sense in the context of relational databases.
\end{abstract}

Keywords: Conceptual modeling; Database; Relational data.

\section{Resumen}

El modelado de datos es el componente principal de la información conceptual en la base de datos, entre las técnicas para este modelado, la entidad-relación (ER) se aborda en este trabajo. El objetivo es abordar la información principal sobre el modelo entidad-relación como herramienta para el modelado conceptual de datos. El Modelo Entidad-Relación, y especialmente el diagrama, es una herramienta importante durante el desarrollo de sistemas, especialmente aquellos que son más complejos y difíciles de visualizar sin mayor análisis. Además, es un modelo conceptual utilizado en Ingeniería de Software para describir las entidades involucradas en un dominio empresarial, con sus atributos y cómo se relacionan entre sí. En general, representa de forma abstracta la estructura que tendrá la base de datos de la aplicación. La base de datos puede contener varias otras entidades, como claves y tablas intermedias, que pueden tener sentido en el contexto de las bases de datos relacionales.

Palabras clave: Base de datos; Datos relacionales; Modelado conceptual. 


\section{Introdução}

Um diagrama entidade relacionamento (ER) é um tipo de fluxograma que ilustra como "entidades", pessoas, objetos ou conceitos, se relacionam entre si dentro de um sistema (Nogueira, 1988). Diagramas ER são mais utilizados para projetar ou depurar bancos de dados relacionais nas áreas de Engenharia de Software, sistemas de informações empresariais, educação e pesquisa (Genong et al., 2010; Kawabata, 2015). Também conhecidos como DERs, ou modelos ER, usam um conjunto definido de símbolos, tais como retângulos, diamantes, ovais e linhas de conexão para representar a interconectividade de entidades, relacionamentos e seus atributos (Sordi et al., 2009; Genong et al., 2010). Eles espelham estruturas gramaticais, onde entidades são substantivos e relacionamentos são verbos (Silva et al., 2008). Diagramas ER estão relacionados com diagramas de estrutura de dados (DEDs), que incidem sobre as relações de elementos dentro de entidades em vez de relações entre as próprias entidades. Diagramas ER são também muitas vezes utilizados junto com diagramas de fluxo de dados (DFDs), que mapeiam o fluxo de informações para processos ou sistemas de dados (Santos et al., 2017).

A modelagem de dados é o principal componente de informações conceituais do banco de dados, dentre as técnicas para essa modelagem, a entidade-relacionamento (ER) apresentada em 1976 por Peter Chen (Chen, 1976; Elmasri \& Navathe, 2005), e ainda largamente pela sua simplicidade e legibilidade, produzindo um modelo que seja inteligível para o desenvolvedor do banco, assim como pelo usuário final (Bagui \& Earp, 1964; Teorey et al., 2006; Silberschatz et al., 2006). É importante mencionar que várias empresas vem adotando o diagrama de classes da UML (Unified Modeling Language) como uma alternativa ao modelo ER. Mesmo que, por um lado, o diagrama de classes tenha tido inspiração no modelo ER e também consiga capturar os requisitos de dados do mundo real de uma maneira simples e significativa produzindo um modelo inteligível, essa não foi a motivação por trás da sua criação (Sommerville, 2003; Roumbaugh et al., 2005). Em razão disso, grande parte das instituições de ensino superior ainda utilizam o modelo ER no ensino de modelagem de dados conceitual. Contudo, nota-se carência quanto a ferramentas de notação de Chen (1976) estendida e que tenham foco no modelo conceitual. Por isso, professores adotaram ferramentas voltadas para o modelo lógico, como exemplos: DBDesigner e ERWin (Silva, 2008; Kazi et al., 2014; Matos, 2016).

Modelar conceitualmente uma realidade é uma das etapas da construção de um Sistema de Informação (Almeida et al., 2010). A modelagem conceitual objetiva a compreensão e representação do mundo real, de tal forma que ele possa ser traduzido em um modelo que capte os aspectos que se deseje estudar de uma realidade. Na ciência da computação um projeto de banco de dados, muitas vezes pode ser uma tarefa difícil por ser necessário que o projetista compreenda as necessidades dos usuários e tenha a capacidade de representá-las. Para isso é necessário que ele consiga entender de forma clara a realidade que está sendo modelada. Muitas vezes o projetista não possui conhecimento sobre o domínio modelado, reforçando a necessidade da interação com o usuário, conhecedor deste domínio. Na fase do projeto em que se modela conceitualmente um banco de dados, o enfoque está na construção de uma representação de alta qualidade dos fenômenos selecionados de domínio (Sommerville, 2003). Neste caso, na modelagem conceitual busca-se a identificação, descrição e entendimento de um domínio, processo que permitirá estruturar os conceitos e as relações, nele inseridas (Maia \& Alvarenga, 2014).

Diante dos pressupostos, o objetivo com esse estudo é abordar as principais informações sobre o modelo entidaderelacionamento como ferramenta para modelagem de dados conceituais aplicados na Engenharia de Software.

\section{Metodologia}

Trata-se de um estudo de levantamento de dados, realizado por meio de consulta a base de dados de periódicos da CAPES, repositórios institucionais e relatórios técnicos. De acordo com Pereira et al. (2018), o levantamento das informações caracteriza-se como sendo do tipo descritivo exploratório, de caráter qualitativo, visando a análise, a comparação e o cruzamento de dados entre diversos artigos e literaturas relacionadas ao tema central do estudo, mais especificamente na área de Sistemas de Informação e Engenharia de Software, assim como realizou Santos et al. (2020). 
A pesquisas e coletas de dados foram realizadas de novembro de 2019 a novembro de 2020, a partir de questões levantadas sobre o tema e foram consultadas cerca de 30 obras por meio de revisões bibliográficas, tendo sido buscados artigos científicos, livros e referências em bancos de dados eletrônicos e nas bases bibliográficas: Google, Google Acadêmico, Amazon e Scielo. Para coleta das informações, foram buscados os seguintes descritores: banco de dados, dados relacionais, Diagrama Entidade-Relacionamento, Engenharia de Software, modelagem conceitual, e outros.

\section{Modelagem Conceitual com o Modelo Entidade-Relacionamento (ER)}

É um modelo popular para modelagem conceitual de dados. Esse modelo e suas variações, como o Modelo EntidadeRelacionamento Estendido, são frequentemente usados para o projeto conceitual de aplicações de banco de dados. As extensões do ER são geralmente usadas na modelagem conceitual de bancos de dados mais complexos, como os bancos de dados científicos (Santos, 2017). O ER se baseia em três conceitos básicos: entidades, atributos e relacionamentos (Ribeiro, 1992; Ali et al., 2010).

Uma entidade representa um elemento do mundo real, como um funcionário ou uma disciplina. Um atributo pode ser: simples ou atômico, quando não pode ser dividido, como nome; composto, que é dividido em várias partes, como endereço, que é dividido em rua, número e CEP; de valor único, que tem um só valor para uma determinada entidade, como idade; multivalorado, que pode assumir vários valores para uma determinada entidade, como telefone; e derivado, que é um atributo que pode ser determinado a partir de outros atributos, como idade, que pode ser calculada a partir da data de nascimento e da data de hoje (Silva, 2008). Um banco de dados pode conter grupos de entidades que são similares, ou seja, compartilham dos mesmos atributos. Contudo, cada entidade tem um valor para cada atributo, esse grupo de entidades similares é chamado de tipo de entidade, sendo que cada tipo de entidade é descrito por seu nome e seus atributos (Moraes, 2015; Muslah \& Ghoul, 2019). Por exemplo, Empregado é um tipo de entidade que contém os atributos CPF, nome, idade e salário.

Todo tipo de entidade tem um ou mais atributos chave, cujos valores são distintos para cada entidade do conjunto de entidades. Portanto, os valores desses atributos podem identificar uma única entidade (Santos, 2017). Para o tipo de entidade Empregado, podemos usar CPF como atributo chave. Os tipos de entidade que não tem atributos chave são chamados de tipos de entidade fraca. Sua chave será determinada por um tipo de relacionamento com uma entidade forte (Panigassi, 2007; Ali et al., 2010). Um relacionamento se dá entre duas ou mais entidades e representa uma associação entre estas, como o relacionamento trabalha entre um funcionário e um projeto. Um conjunto de associações ou relacionamentos entre entidades é definido como um tipo de relacionamento entre tipos de entidade. Todo tipo de relacionamento contém um grau, que indica o número de tipos de entidade que participam desse relacionamento (Moraes, 2015; Muslah \& Ghoul, 2019).

Os tipos de relacionamento podem conter atributos, por exemplo, o tipo de relacionamento em atribuir horas para indicar quantas horas cada empregado trabalha em um departamento. Os tipos de relacionamento de grau dois, ou binários, apresentam dois tipos de restrições: a de cardinalidade e a de participação. A cardinalidade de um relacionamento especifica o número máximo de instâncias do relacionamento em que uma entidade pode participar (Kazi et al., 2014; Matos, 2016). Por exemplo, o tipo de relacionamento entre empregado e departamento tem cardinalidade $\mathrm{N}: 1$, o que significa que cada empregado pode trabalhar em somente um departamento, enquanto que em cada departamento podem trabalhar inúmeros empregados. As cardinalidades possíveis são: 1:1, 1:N, N:1 e M:N (Maia \& Alvarenga, 2014).

Existem dois tipos de participação, a total e a parcial. Se considerarmos que todo empregado deve trabalhar em um departamento, então a participação de empregado no tipo de relacionamento é total. No entanto, no tipo de relacionamento gerenciado, pode-se dizer que a participação de empregado é parcial, pois nem todo empregado gerencia um departamento. Os diagramas ER são utilizados como uma notação diagramática associada ao modelo de ER (Silva, 2008). 


\subsection{O Modelo Entidade-Relacionamento Estendido (EER)}

O modelo introduz construtores adicionais que possibilitam a representação de conceitos como o de especialização, generalização e categorização. Especialização é o processo de definir um conjunto de subclasses de um tipo de entidade; esse tipo de entidade é chamado de superclasse da especialização (Maia \& Alvarenga, 2014). Por exemplo, Secretária, Engenheiro e Médico são subclasses de Empregado. Generalização é o processo reverso da especialização, ou seja, as características comuns de diferentes tipos de entidades são identificadas e generalizadas em uma única superclasse (Moraes, 2015; Muslah \& Ghoul, 2019).

Existem dois tipos de restrições aplicada à especialização: de disjunção e de completude. A restrição de disjunção especifica que as subclasses da especialização devem ser disjuntas, ou seja, uma entidade pode ser membro de no máximo uma das subclasses da especialização (Santos, 2017). Uma especialização disjunta é representada com um "d" dentro do círculo. Se as subclasses não forem disjuntas, então elas são sobrepostas e a especialização sobreposta será representada com um "o" dentro do círculo. A restrição de completude pode ser total ou parcial (Sordi et al., 2009; Genong et al., 2010). Uma especialização total indica que toda entidade da superclasse deve ser membro de pelo menos uma das subclasses e é representada pela linha dupla que liga a superclasse ao círculo. Quando a especialização é parcial ela é representada com uma linha única e permite que uma entidade não pertença a nenhuma das subclasses (Kazi et al., 2014; Matos, 2016). Geralmente, a superclasse identificada na generalização é total, pois ela é derivada das subclasses e por isso contém apenas entidades que estão nas subclasses.

Na categorização, uma subclasse, chamada categoria, vai representar uma coleção de objetos que é um subconjunto da união de tipos de entidades distintos (Santos, 2017). Por exemplo, em um banco de dados para registro de veículos, um dono de um veículo pode ser uma pessoa, um banco ou uma empresa. As superclasses Empresa, Banco e Pessoa são conectadas ao círculo com o símbolo U, que representa a operação de união de conjuntos. Uma categoria pode ser total ou parcial. Uma categoria total contém a união de todas as entidades em suas superclasses, enquanto que uma categoria parcial pode conter um subconjunto da união (Panigassi, 2007).

\subsection{Importância do Diagrama Entidade-Relacionamento}

Sabe-se da importância dos bancos de dados. Em diversas áreas pode ser necessário o armazenamento de informações. $\mathrm{Na}$ área de saúde, por exemplo, pode-se desejar armazenar informações diversas sobre funcionários, pacientes, atendimentos, tratamentos para determinadas doenças, etc (Panigassi, 2007). Da mesma forma, em um sistema bancário, há um grande volume de dados armazenado a respeito dos correntistas, suas contas e transações efetuadas (Kazi et al., 2014; Matos, 2016).

Independentemente da área, percebe-se a necessidade de armazenar e manipular dados e neste contexto é fundamental que estes sejam armazenados de forma organizada e permitam um posterior acesso eficiente. Neste sentido, torna-se necessário projetar os bancos de dados (Moraes, 2015; Muslah \& Ghoul, 2019). O Diagrama Entidade-Relacionamento utiliza elementos gráficos para descrever o modelo de dados de um sistema com alto nível de abstração. O diagrama parte do princípio que o mundo real é formado por um conjunto de objetos chamados entidades e por relacionamentos entre esses objetos (Silberschatz et al., 1999). Navathe e Elmasri (2005) sugerem a notação apresentada na Figura 1 como base para um DER. 
Figura 1 - Notação sugerida para um Diagrama Entidade-Relacionamento.

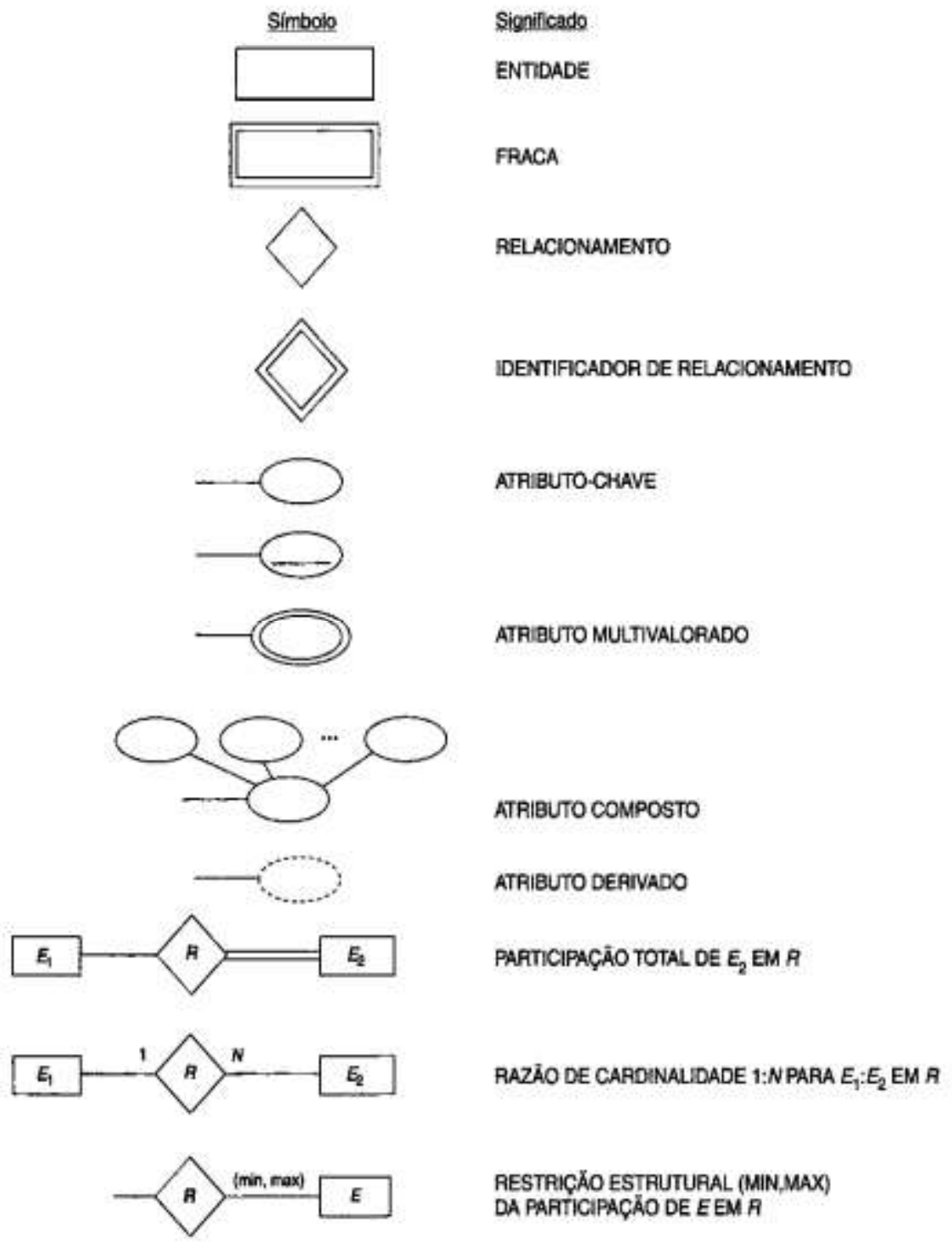

Fonte: Elmasri \& Navathe (2005).

Tendo como base os elementos gráficos exemplificados na Figura 1, pode ser criado o Diagrama EntidadeRelacionamento apresentado na Figura 2. 
Figura 2 - Exemplo de um DER.

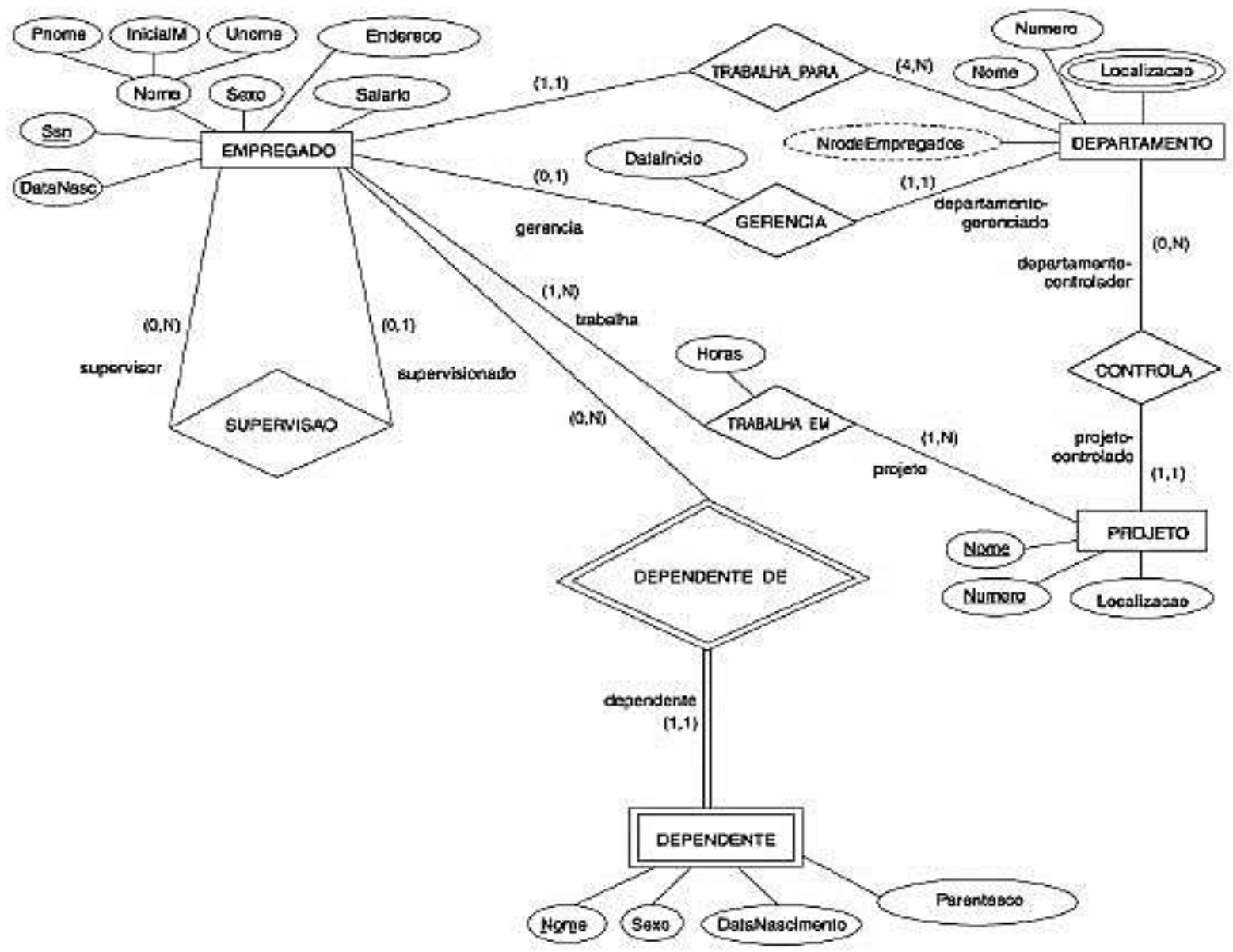

Fonte: Navathe \& Elmasri (2005).

O diagrama é composto de entidades, relacionamentos, seus atributos e cardinalidades, como visto no item 3.1 deste trabalho. As entidades representam um objeto ou conceito da realidade e os relacionamentos exibem qual a ligação entre elas, utilizando das cardinalidades para definir a sua interação. Como pode ser observado na Figura 2, a entidade "Empregado" está relacionada com ela mesma e com as entidades "Dependente", "Projeto" e "Departamento".

Entidades são representadas graficamente por retângulos, e possuem atributos, que são responsáveis por caracterizá-las, fazendo referência a dados como nome, sexo, telefone, endereço, etc. $\mathrm{O}$ atributo definido para representar a entidade é chamado de "Chave-Primária" (Santos, 2017). Uma entidade pode ser definida como fraca quando seu campo identificador depende diretamente de outra entidade, não tendo sua própria chave. Na Figura 2, a entidade "Dependente" é um exemplo de entidade fraca em relação à entidade "Empregado", sendo representada graficamente por um segundo retângulo ao seu redor. Certo dependente só existirá caso haja um empregado.

De acordo com Navathe e Elmasri (2005) podem existir diversos tipos de atributos em determinado diagrama: simples versus composto, monovalorado versus multivalorados, armazenado versus derivado. Os atributos compostos podem ser divididos em subclasses, como no caso de um atributo "Endereço", que pode ser composto pelos atributos "Rua", "Numero", "Bairro" e "Cidade", por exemplo. Na Figura 2, a entidade "Empregado" possui um atributo composto do tipo "nome". Quanto a atributos multivalorados Navathe e Elmasri (2005) citam o exemplo de um atributo para representar a titulação de uma pessoa, afinal, pessoas distintas podem possuir diferentes números de titulações, que pode ser nenhuma, uma ou várias. No entanto, 
atributos derivados são aqueles que farão referência a um atributo armazenado, como no caso de data de nascimento (armazenado) e idade (derivado). O atributo idade irá variar de acordo com o atributo data de nascimento.

Date (2004), cita que um relacionamento mostra a forma de comunicação entre determinadas entidades. Os relacionamentos são representados por losangos. Quanto à cardinalidade, Takai et al. (2005) resumem como sendo a quantidade de instâncias de relacionamento que uma entidade pode participar. Ou seja, quantas vezes certa entidade poderá se relacionar com outra. Por exemplo, na Figura 2 existe o relacionamento "Controla" entre as entidades "Departamento" e "Projeto", com suas cardinalidades $(0, \mathrm{~N})$ e $(1,1)$ respectivamente. Isto significa que cada departamento pode controlar nenhum ou vários projetos, e cada projeto é controlado somente por um departamento.

Cabe ressaltar que a elaboração de um diagrama, como mostrado na Figura 2, não é uma tarefa simples. Para que uma modelagem seja bem feita e consiga representar adequadamente os requisitos relacionados é preciso um entendimento claro de todos os requisitos além de um estudo completo sobre a elaboração de diagramas entidade relacionamento (Santos, 2017).

\section{Aplicação da Ferramenta em Sistema Imobiliário}

Enquanto o MER é um modelo conceitual, o Diagrama Entidade Relacionamento (Diagrama ER ou ainda DER) é a sua representação gráfica e principal ferramenta. Em situações práticas, o diagrama é tido muitas vezes como sinônimo de modelo, uma vez que sem uma forma de visualizar as informações, o modelo pode ficar abstrato demais para auxiliar no desenvolvimento do sistema (Silva, 2008; Qian, 2013). Dessa forma, quando se está modelando um domínio, o mais comum é já criar sua representação gráfica, seguindo algumas regras.

O diagrama facilita ainda a comunicação entre os integrantes da equipe, pois oferece uma linguagem comum utilizada tanto pelo analista, responsável por levantar os requisitos, e os desenvolvedores, responsáveis por implementar aquilo que foi modelado (Qian, 2013; Santos, 2017). Em sua notação original, proposta por Chen (1976), as entidades deveriam ser representadas por retângulos, seus atributos por elipses e os relacionamentos por losangos, ligados às entidades por linhas, contendo também sua cardinalidade $(1 . .1,1 . . n$ ou n..n). Porém, notações mais modernas abandonaram o uso de elipses para atributos e passaram a utilizar o formato mais utilizado na UML, em que os atributos já aparecem listados na própria entidade. Essa forma torna o diagrama mais limpo e fácil de ser lido. Um exemplo simples de um diagrama para um sistema de imobiliárias (Figura 3).

Figura 3 - Diagrama Entidade-Relacionamento de sistema de imobiliária.

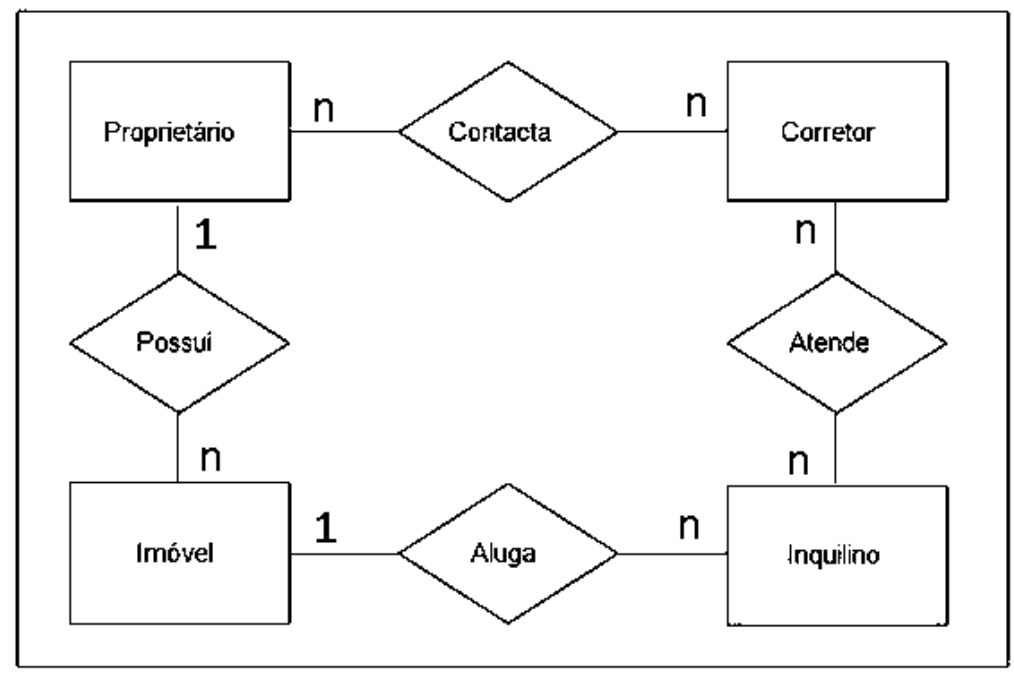

Fonte: Vilas-Boas (2001). 
No domínio representado pelo diagrama acima temos as seguintes entidades e relacionamentos:

- Proprietário contata Corretor (um proprietário pode contatar vários corretores e um corretor pode ser contatado por vários proprietários).

- Corretor atende Inquilino (um corretor pode atender vários inquilinos e um inquilino pode ser atendido por vários corretores).

- Inquilino aluga Imóvel (um inquilino aluga um imóvel e um imóvel pode ser alugado por vários inquilinos).

- Proprietário possui Imóvel (um proprietário possui vários imóveis e um imóvel pertence a apenas um proprietário).

Uma variante pode ser apresentada, onde a cardinalidade do relacionamento é exibida junto a um losango (Figura 4 e

$5)$.

Figura 4 - Diagrama de Entidade Relacionamento (variação 1).

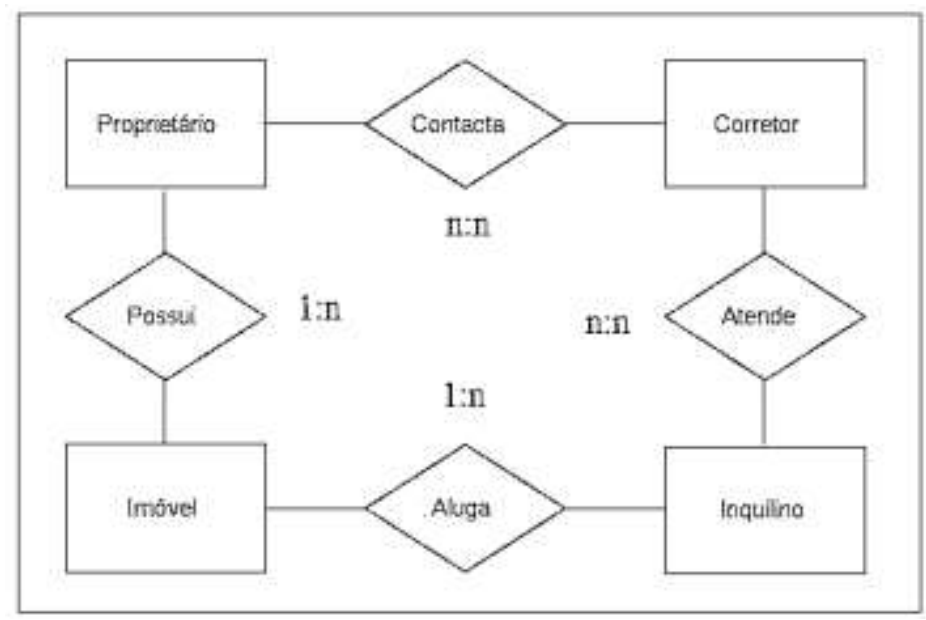

Fonte: Vilas-Boas (2001).

Uma outra variação mostra a cardinalidade de uma forma mais completa, deixando claro as possibilidades de números de objetos envolvidos em cada relacionamento (Nogueira, 1988). Nesse modelo, em cada lado do relacionamento os números aparecem no formato $(\mathrm{X}, \mathrm{Y})$ ao invés de um único número como vemos nas figuras anteriores. A Figura 5 ilustra um exemplo desse tipo.

Figura 5 - Diagrama Entidade Relacionamento (variação 2).

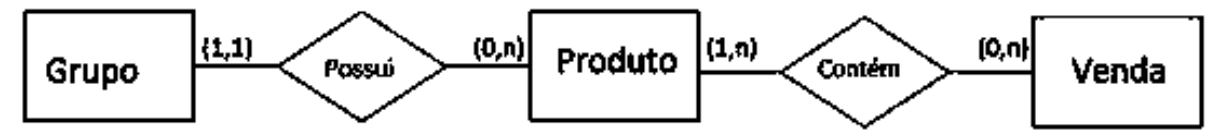

Fonte: Nogueira (1988).

Os atributos podem aparecer no diagrama na forma de elipses ligadas às entidades. Essa foi a notação original proposta, mas como pode-se observar na Figura 6, o modelo deixa o diagrama com muitos itens e pode atrapalhar a organização do sistema conceitual. 
Figura 6 - Atributos apresentados como elipses.

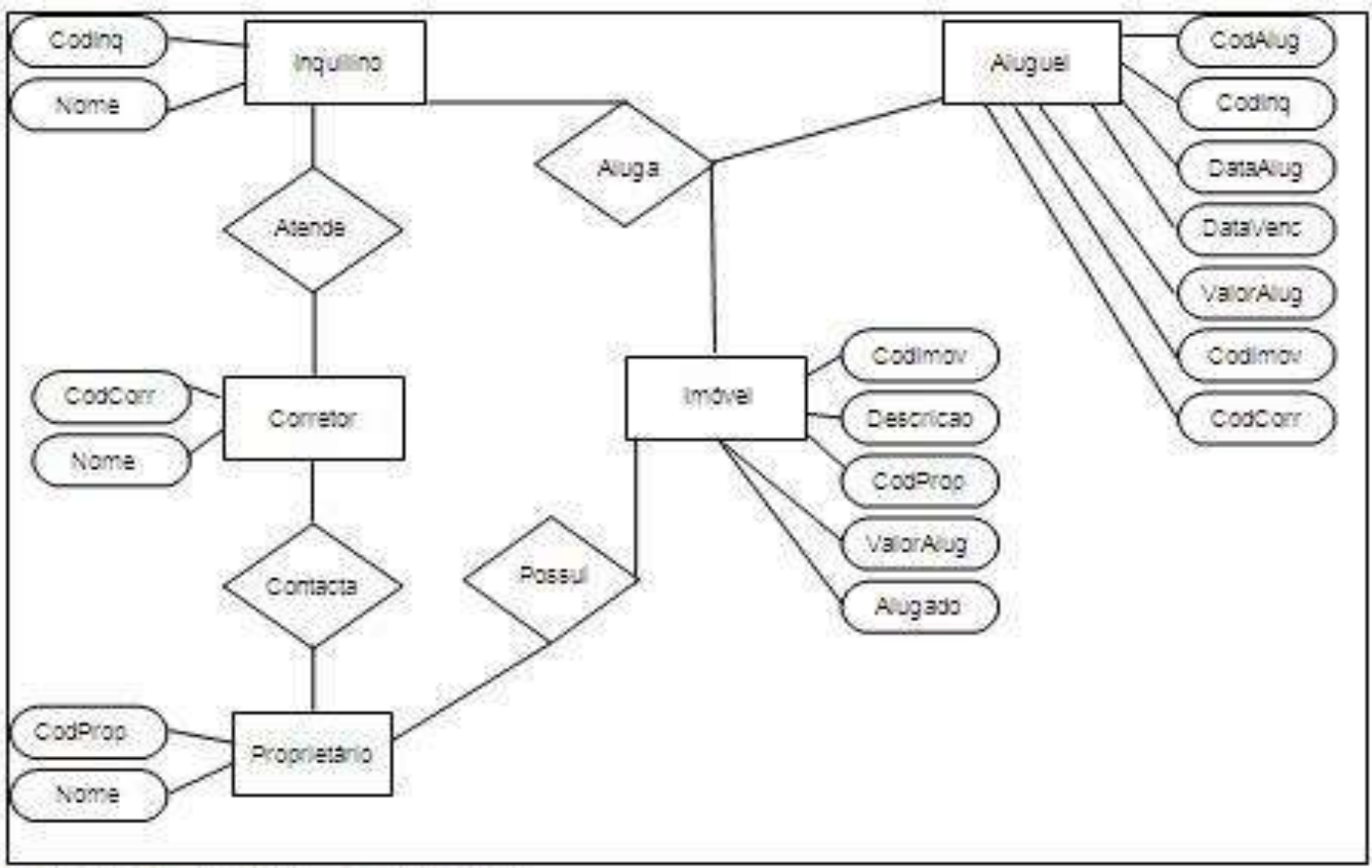

Fonte: Nogueira (1988).

Em uma notação mais atual, comumente utilizada na UML, os atributos aparecem listados dentro do próprio retângulo da entidade, enquanto o nome da entidade aparece no topo na forma de título (Figura 7).

Figura 7 - Diagrama com atributos nas entidades.

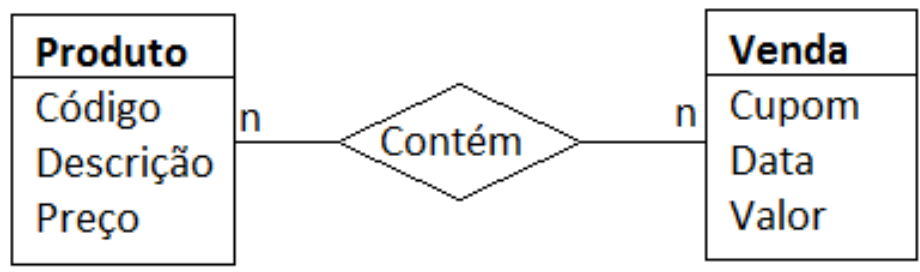

Fonte: Vilas-Boas (2001).

\subsection{Exemplo prático}

Para ementar o conteúdo desse trabalho, apresenta-se um pequeno exemplo prático em que se modelou um sistema de bibliotecas, focando especificamente no empréstimo de livros, baseando-se nos esquemas desenvolvidos por Almeida et al. (2010) e Santos (2017). A priori identificaram-se as entidades envolvidas nesse contexto. Sabe-se que as entidades físicas existentes são o Usuário da biblioteca e o Livro que será emprestado. Além disso, consideraremos aqui que o livro pertence a uma Sessão, que ajuda na organização das obras do acervo. Em um sistema real pode haver outras informações sobre o livro, mas para esse exemplo a sessão é o bastante. Por fim, tem-se a entidade lógica Empréstimo, que tanto está relacionada com o usuário, quanto com o livro. Sendo assim, pode-se esboçar um diagrama simples, contendo as principais entidades e o relacionamento entre elas (Figura 8). 
Figura 8 - DER de um sistema para biblioteca.

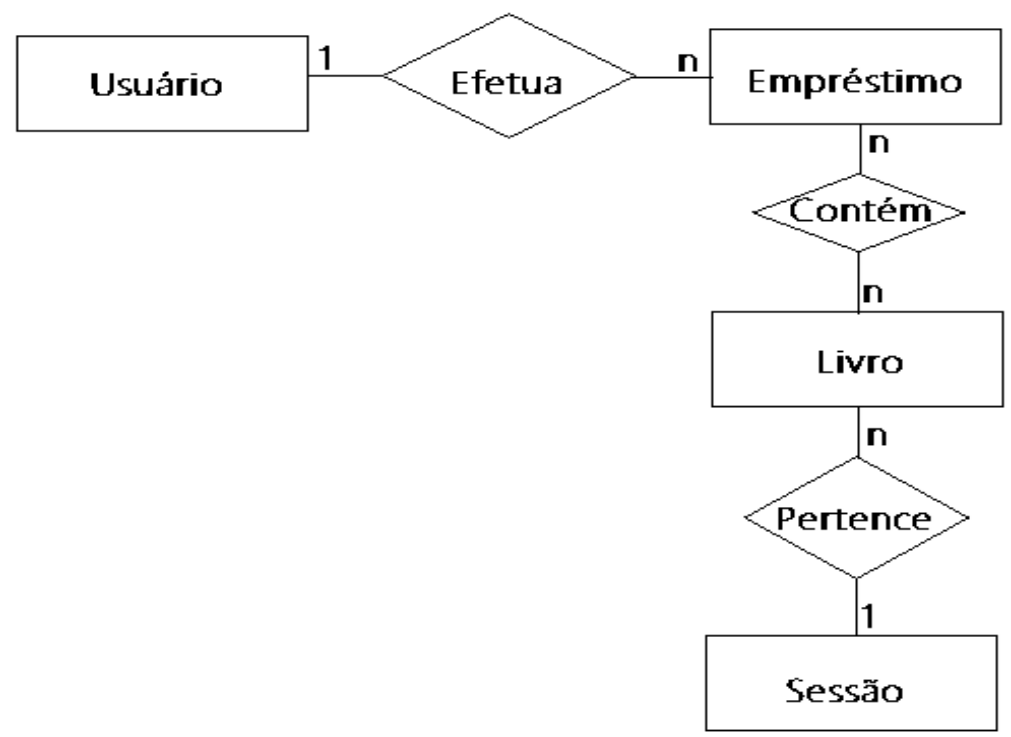

Fonte: Vilas-Boas (2001)

Neste primeiro diagrama podemos identificar alguns dos conceitos vistos:

- Entidades fortes: Usuário, Livro e Sessão;

- Entidades fracas: Empréstimo;

- Relacionamentos: um Usuário efetua vários Empréstimos, vários Empréstimos contêm vários Livros, vários Livros pertencem a uma Sessão.

Baseando-se nos modelos conceituais preparados por Silva (2008). Exemplifica-se o domínio no diagrama, pode-se adicionar os atributos e outras entidades que se façam necessárias (Figura 9).

Figura 9 - DER mais completo do sistema para bibliotecas.

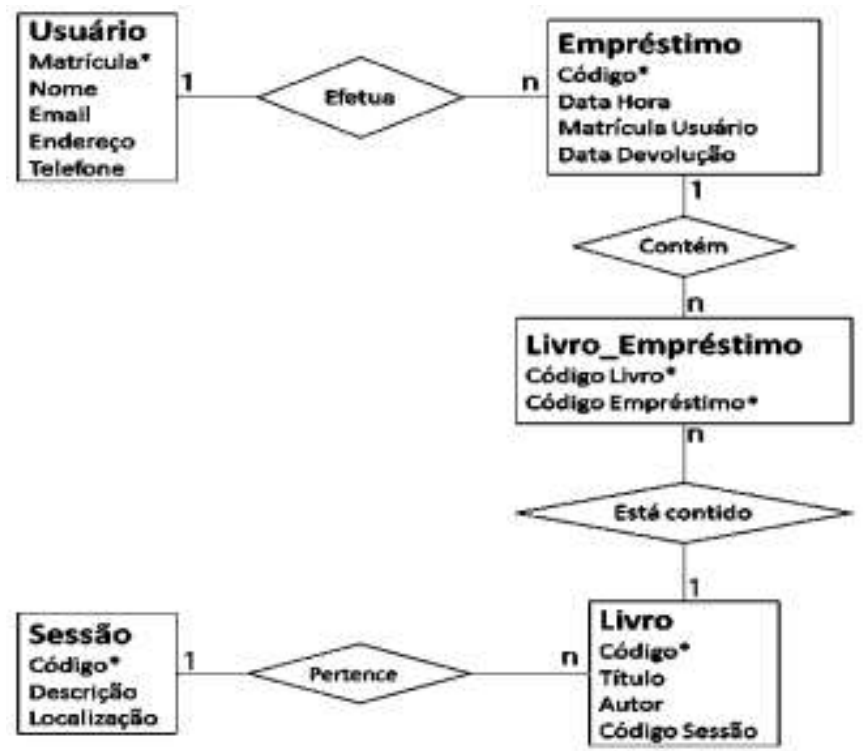

Fonte: Silva (2008). 
De acordo com Fidalgo et al. (2012), especifica-se os atributos de cada entidade e marca-se algumas delas com um asterisco, indicando que é a chave primária da tabela, ou seja, um atributo único, que nunca poderá se repetir entre as entidades do mesmo tipo. Pode-se notar que neste momento ainda não é necessário especificar o tipo de cada atributo (texto, número, data, etc.), isso só será necessário mais adiante, quando já estiver planejando o banco de dados da aplicação. Surgiu a entidade associativa Livro-Empréstimo, que representa os livros contidos em um empréstimo, considerando um empréstimo contém vários livros e um livro pode estar contido em vários empréstimos. Conforme Fidalgo et al. (2013), esta entidade é composta pelas chaves das duas entidades principais. Se fosse necessário, nesta entidade também pode-se adicionar informações complementares como quantidade, não se aplica neste caso, mas caberia em um sistema de vendas, por exemplo.

E mais, segundo Fidalgo et al. (2012) e Fidalgo et al. (2013), na entidade associativa, o relacionamento n..n foi dividido em dois relacionamentos do tipo 1..n, agora lidos da seguinte forma: um empréstimo contém vários itens, mas um item só pode estar contido em um único empréstimo (restrito pelas chaves primárias); um livro pode estar contido em vários itens de empréstimo (ser emprestado várias vezes), mas cada item refere-se a um único livro (Silva, 2008). A correta modelagem auxilia no correto desenvolvimento da base de dados e evita que várias alterações sejam necessárias para corrigir erros de concepção provenientes de falhas durante a análise, ou ainda por problemas de comunicação entre os membros da equipe (Fidalgo et al., 2013).

\section{Considerações Finais}

O Modelo Entidade-Relacionamento, e principalmente o diagrama, é uma importante ferramenta durante o desenvolvimento de sistemas, principalmente aqueles mais complexos e difíceis de visualizar sem uma análise mais aprofundada. Além disso, é um modelo conceitual utilizado na Engenharia de Software para descrever as entidades envolvidas em um domínio de negócios, com seus atributos e como elas se relacionam entre si. De modo geral, representa de forma abstrata a estrutura que possuirá o banco de dados da aplicação. O banco de dados poderá conter várias outras entidades, tais como chaves e tabelas intermediárias, que podem fazer sentido no contexto de bases de dados relacionais.

Entretanto, sempre serão criados modelos para um sistema completo, porque isso poderia resultar em um modelo muito extenso e difícil de interpretar. Dependendo da magnitude do que estaremos desenvolvendo, podemos criar modelos apenas para uma parte do sistema, um módulo, ou mesmo uma funcionalidade. O modelo supracitado contempla vendas, finanças, recursos humanos, etc. Várias entidades estão presentes em mais de uma parte do sistema, mas não seria muito interessante, e provavelmente nem mesmo necessário, criar um único modelo para todo o sistema, por isso pode-se dividir a modelagem em várias partes menores para modelagem de dados conceituais.

\section{Referências}

Ali, A., Qadri, S., Muhammad, S. S., Abbas, J., Pervaiz, M. T. \& Awan, S. (2010). Software Cost Estimation through Entity Relationship Model. Journal of American Science, 6(11): 47-51.

Almeida, M. B., Oliveira, V. N. P. \& Coelho, K. C. (2010). Estudo Exploratório sobre Ontologias Aplicadas a Modelos de Sistemas de Informação: Perspectivas de Pesquisa em Ciência da Informação. Encontros Bibli, 15(30): 32-56.

Bagui, S. \& Earp, R. (1964). Database Design Using Entity-Relationship Diagrams. Berker: CRC Press LLC. 321p.

Chen, P. P. (1976). The entity-relationship model: towards a unified view of data. ACM Trans. Database System, p.9-36.

Date, C. J. (2004). Introdução ao Sistema de Banco de Dados. 8.ed. São Paulo: Campus. 896p.

Elmasri, R. \& Navathe, S. B. (2005). Sistemas de Banco de Dados. 4.ed. Florianópolis: Pearson Education do Brasil Ltda.

Fidalgo, N. R., Souza, E. M., España, S., Castro, J. B. \& Pastor, O. (2012). EERMM: Um Metamodelo para o Modelo Aprimorado de EntidadeRelacionamento. Lecture Notes in Computer Science, 515-524. doi: 10.1007/978-3-642-34002-4_40 
Fidalgo, N. R., Alves, E., España, S., Castro, J. \& Pastor, O. (2013). Metamodeling the Enhanced Entity-Relationship Model. Journal of Information and Data Management, 4(3): 406-420.

Genong, Y., Liping, D., Zhang, B. \& Wang, H. (2010). Coordination Through Geospatial Web Service Workflow in the Sensor Web Environment. IEEE Journal of Selected Topics in Applied Earth Observations and Remote Sensing, 3(4): 433-441.

Kawabata, T. (2015). Deteç̧ão de outliers espaciais: refinamento de similaridade e desempenho. 79 p. Dissertação (Mestrado em Ciência da Computação) Universidade Estadual Paulista “Julio de Mesquita Filho”, São José do Rio Preto, SP.

Kazi, L., Radulovic, B., Berkovic, I. \& Kazi, Z. (2014). Integration of conceptual data modeling methods: Higher education experiences. 201437 th International Convention on Information and Communication Technology, Electronics and Microelectronics (MIPRO). doi:10.1109/mipro.2014.6859685

Maia, R. M. C. \& Alvarenga, L. (2014). Teoria da Classificação Facetada e Contribuições para o Modelo Entidade Relacionamento. Revista Prisma, 25: 91-125.

Matos, H. G. (2016). Uma avaliação sobre os métodos para modelagem conceitual de banco de dados. 118p. Dissertação (Mestrado em Ciência da Computação) - Universidade Federal de Pernambuco, Recife, PE.

Moraes, L. C. (2015). Representação de variabilidade estrutural de dados por meio de famílias de esquemas de banco de dados. 47p. Dissertação (Mestrado em Ciência da Computação) - Universidade de São Paulo, São Paulo, SP.

Muslah, E. \& Ghoul, S. (2019). Requirements variability specification for data intensive software. International Journal of Software Engineering \& Applications, $10(2)$.

Nogueira, D. L. (1988). Ferramentas automatizadas para apoio ao projeto estruturado: uma aplicação do diagrama de entidade-relacionamento. 336f. Tese (Doutorado em Ciências em Engenharia de Sistemas e Computação) - Universidade Federal do Rio de Janeiro, Rio de Janeiro, RS.

Panigassi, R. (2007). Método para especificação e modelagem de processos de fábrica de software usando RM-DOP e BPM. 152p. Dissertação (Mestrado em Engenharia) - Universidade de São Paulo, São Paulo, SP.

Pereira, A. S., Shitsuka, D. M., Parreira, F. J. \& Shitsuka, R. (2018). Metodologia da pesquisa científica. 1.ed. Santa Maria, RS: UFSM, NTE.

Qian, R. (2013). The Application of GIS in the Real Estate Management System. In: Du, Z. (eds). Proceedings of the 2012 International Conference of Modern Computer Science and Applications. Advances in Intelligent Systems and Computing, 191. Springer, Berlin, Heidelberg. https://doi.org/10.1007/978-3-64233030-8_90

Ribeiro, C. D. (1992). Um gerador de transações voltado para o modelo entidade-relacionamento. 119f. Tese (Doutorado em Ciências em Engenharia de Sistemas e Computação) - Universidade Federal do Rio de Janeiro, Rio de Janeiro, RJ.

Rumbaugh, J., Jacobson, I. \& Booch, G. (2005). The Unified Modeling Language Reference Manual. 2.ed. São Paulo: AddisonWesley Professional. 496p.

Santos, P. H. (2017). Diagrama ER é uma ferramenta voltada ao ensino de modelagem de dados. 44p. Dissertação (Mestrado em Ciências da Computação) Universidade Federal de Minas Gerais, Belo Horizonte, MG.

Santos, S. H. N., Costa, Y. J. S., Santos, D. V., Barradas Filho, A. O., Bottentuit Junior, J. B. \& Cabrejos, L. J. E. R. (2020). Identifying serious games for software engineering teaching in brazil through a systematic mapping study. Research, Society and Development, 9(7):1-22. e329973702.

Silberschatz, A., Horth, H. F. \& Sudarshan, S. (2006). Sistema de Banco de Dados. São Paulo: Pearson. 223p.

Silva, W. C. (2008). Uma ferramenta para geração automática de modelo conceitual de classes baseada em processamento de linguagem natural. 2008. 117p. Dissertação (Mestrado em Ciência da Computação) - Universidade Metodista de Piracicaba, Piracicaba, SP.

Sommerville, I. (2003). Engenharia de software. 5.ed. São Paulo: Pearson. 592p.

Sordi, J. O., Picchiai, D., Costa, M. A. M. \& Sanches, M. A. (2009). Competências críticas ao desenvolvimento de mapas cognitivos de redes interorganizacionais. Revista de Administração Pública, 43(5): 1181-1206.

Takai, O. K., Italiano, I. S. \& Ferreira, J. E. (2005). Introdução à Banco De Dados. São Paulo: IME: USP. 124p.

Teorey, T., Lighstone, S. \& Nadeau, T. (2007). Projeto e Modelagem de Banco de Dados. São Paulo: Elsevier.

Vilas-Boas, J. H. (2001). Bases teóricas e metodológicas, da abordagem geográfica do ordenamento territorial, aplicada para o desenvolvimento de sistema de banco de dados georreferenciáveis: exemplo da bacia do Rio Tapecuru. 292 f. Tese (Doutorado em Geografia) - Universidade de São Paulo, São Paulo, SP. 